\title{
How and Where the Standard Model of Particle Physics Hides Dark Matter
}

\section{Housam H Safadi}

Safadi Bureau Damascus, Syria,

\begin{abstract}
The Standard Model of particle physics is thought to be the best map that describes our life. For this reason, it could embed dark matter and reason gravity. In this exploration, I am looking at Standard Model through a new approach different from merely classifying particles as fermions and bosons. I will search in them for the concept and role of massiveness. Specifying photons and gluons as the unique bosons declared in Standard Model, I go looking for revealing the secrets of Higgs particle, $\mathrm{Z}$ and $\mathrm{W}$-, which should not be visible matter bosons.
\end{abstract}

Corresponding author: Housam H. Safadi, Msc.Eng., Safadi Bureau Damascus, Syria, Phone: 5120197 \& 5123493, E-mail: hsafadi@gmail.com

Received: March 04, 2020; Accepted: March 11, 2020; Published: March 16, 2020

Keywords: Standard Mode of Particle Pysiics, Dark Matter; Higgs Particle Entity, Z; W, Applied Social Dark Matter Science, Old Testament or OT New Testament or NT, Qur 'an'.

\section{New Approach Seeing Weak Force}

My simple instinct engineering approach refuses force and mass unity in Higgs particle, $\mathrm{Z}$ and $\mathrm{W}$ of Standard Model. Higgs particle, $Z$ and $W$ either massive particle in the visible universe or massless boson as others, not both. Engineering intuition and faith forced me to look for revealing this puzzle. Massive particles in the Standard Model are quarks and electrons, which form the known atoms of visible matter. We will not regard boson to other massive particles, which are Z, W and Higgs particle. Standard Model particles' properties help us dividing them into two categories. The first are quarks and electrons, both are L-R handed particles. They form the known atoms of the periodical table of ordinary, visible matter elements. (Fig. 1). The second covers those Left-handed, which interact only with other left-handed and appear as a ghost in all their interaction activities. These particles are neutrinos, Higgs boson, Z and W-. (Fig. 2-1), (Fig.2-2)

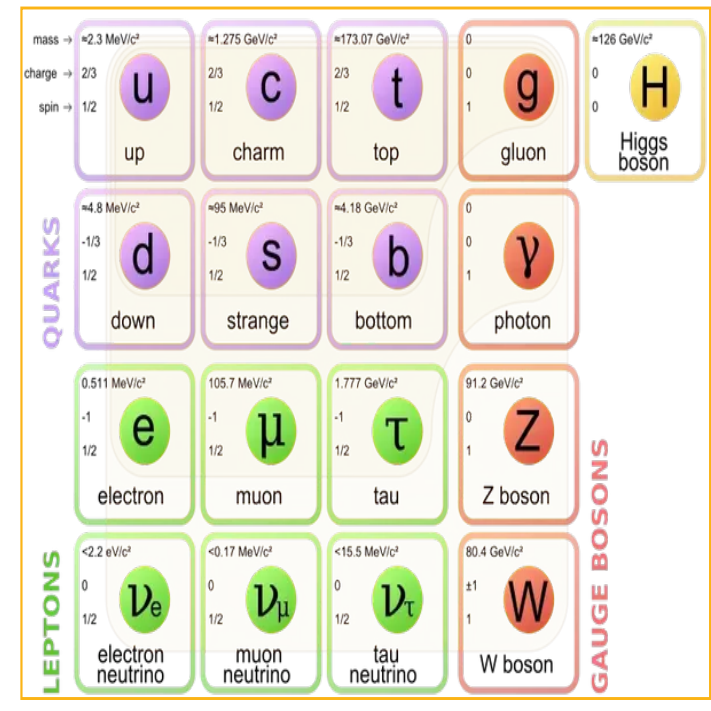

Figure1: Standard Model Particles 


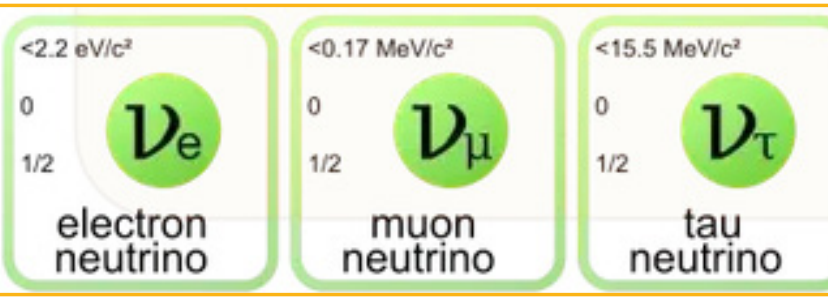

Figure 2-1: Elementary Particleof Dark invisible Matter

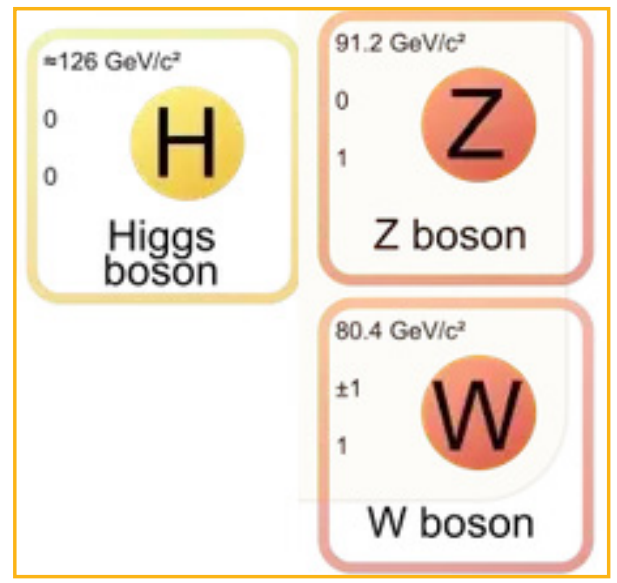

Figure 2-2: Dark Invisible Matter Entities that Interact with immaterial emotional life.

Other Dark Matter Entities like WIMPs, axions, etc.

Figure 2-3: Dark Invisible Matter Entities, Many might be discovered.

Quarks and electrons should have been studied deeply and in detail, while the other massive particles (Neutrinos, Higgs boson, Z, W-) are still disorganized. We will suggest them as parts of dark invisible matter entities, where no place for two kinds of particles, bosons and fermions. Studying the nature of the above nominated invisible dark matter stuff (Neutrinos, Higgs boson, Z, W), imposes defining their elementary particle. We suggest, should be the lightest i.e. neutrino. (Fig. 2-1). Other entities of the above nominated invisible matter stuff, unlike visible matter, are formed of a large amount of one primitive element "neutrino".W- = has $4^{\star} 10^{10}$ neutrinos, $Z=$ carry $4.5^{\star} 10^{10}$ neutrinos, Higgs boson= embed $6^{\star} 10^{10}$ neutrinos. There might be other entities formed from large numbers of neutrinos like axioms, WIMPs, and others. A special table like the known periodical table of visible matter could be organized in future for dark matter entities (Figure. 2-3).

\section{Weak Force Function with Our Living System}

Life and nature constitute two kinds of substances, living and dead. Dead substance shows in many forms: gas, liquid, etc. They are all elements of visible matter periodical table. Living stuff and mainly we humans, are formed from two units, first one material and contains the elements of visible matter of periodical table mainly $\mathrm{C}, \mathrm{H}, \mathrm{O}, \mathrm{N}$ and second involve immaterial invisible stuff, we can refer to as; consciousness, conscience, feelings, appetencies, instincts etc. I realized that the suggested invisible Left-handed particles of the Standard Model (Higgs boson, Z, W-), can interact with the invisible side of our existence; consciousness, conscience, feelings, appetencies, instincts etc $[1,2]$. Higgs boson, Z, and W- role in the current Standard Model is thought to be single as mediators and not part of the interaction. W- role in physics is also believed to be indicated only for agitating radiations [3]. According to our study this might be not the whole story. Our search sees Z, W and Higgs particle (boson) part of the interaction inputs and outputs of invisible dark matter. These invisible particles' interactions are different from those of visible matter particles, simply because their mass is invisible. I think that is the reason why we named them in Standard model bosons though they have mass. I would like to look for a new branch "Applied Social Dark Matter Science". Others perceive that "Neutrino Physics" [4]. This science involves the suggested dark immaterial invisible entities (Higgs boson, Z, W), with our emotional-spiritual life. (Consciousness, conscience, feelings, appetencies, instincts). Higgs particle as uncharged and zero spin could and should go with our living social immaterial environment. Higgs particle might at some level crack to $\mathrm{W}$-, diffusing wield electrons into our neuro system. That might cause hate, religious extremism, murder, raping, stealing, counterfeiting, faking, etc. It is an appropriate time for revising the Standard Model by applying the suggested dark matter stuff of this search (Higgs particle, Z, and W-) to our unseen but realized life social and immaterial existence. These bizarre lost concepts of dark matter entities and activities in Standard Model, may lead my research to be accepted if looked after seriously and subjected to bioengineering experiments [5].

\section{References}

1. Charles S Cokell (2018) The equations of Life, Bacic Books, New York

2. Sean Carroll (2018) The Big Picture. DUTTON-est 1851

3. Wikipedia. Beta particle

4. Wikipedia. Neutrino

5. Wilczek F (2015) A Beautiful Question: Finding Nature 
Citation: Housam H. Safadi (2020) How and Where the Standard Model of Particle Physics Hides Dark Matter. Journal of Physics \& Optics Sciences. SRC/JPSOS/113. DOI: doi.org/10.47363/JPSOS/2020(2)108

Deep Design. PENGUIN Press.

Copyright: (C2020 Housam H. Safadi. This is an open-access article distributed under the terms of the Creative Commons Attribution License, which permits unrestricted use, distribution, and reproduction in any medium, provided the original author and source are credited. 